\title{
Pain Management: Essential Topics for Examinations
}

\author{
Rajesh Gupta. Springer, 2014. ISBN 878-3-642-55060-7
}

\author{
Mehdi Sefidgar, MD
}

Received: 16 January 2015/ Accepted: 30 January 2015/Published online: 6 March 2015

(C) Canadian Anesthesiologists' Society 2015

The study of pain management, whether in the acute or chronic setting, is certainly a daunting task. With the wide multitude of potential diagnoses and the myriad of available treatment options, many of which are for offlabel use, it can be quite challenging to navigate the literature and form concise clear recommendations that can be summarized in a brief text.

This is the task that Dr. Rajesh Gupta undertook when writing Pain Management: Essential Topics for Examination. This text comprises 52 short chapters with a logical progression through anatomy, physiology, medications, and pathophysiology. The first half of the text focuses on imparting the basic knowledge of anatomy and physiology, with individual chapters allocated for each topic. Additionally, this half of the text includes chapters that focus on particular classes of drugs relevant to the practicing pain physician. In the second half of the text, individual chapters are devoted to a wide range of acute and chronic pain syndromes.

The text certainly delivers on its promise of concise information covering a wide range of topics. With only 122 pages, it can be read within a short duration of time, particularly by those already initiated in the field of pain management. Dr. Gupta certainly succeeds in his goal to gather the breadth of information necessary to impart the most knowledge in as brief a format as possible. This is an excellent resource for those unversed in the discipline of pain management, particularly medical students or junior residents. The topics covered here provide an adequate foundation upon which to build.

M. Sefidgar, MD ( $\varangle)$

University of Manitoba, Winnipeg, MB, Canada

e-mail: mehdi.sefidgar@gmail.com
The chapters pertaining to the physiology of pain are excellent, particularly the chapters that focus on pain processing. The models of pain processing are explained in significant detail, including the actions at $\mathrm{Na}, \mathrm{K}, \mathrm{Ca}$, serotonin, and GABAergic receptors, as well as both peripheral and central modulation, with individual chapters allocated for each topic. Furthermore, chapter 3, which focuses on how acute nociception can develop into chronic pain and the pathways involved, is one of the best written explanations on the topic. It is simultaneously concise while still covering the necessary information.

The chapters detailing medications pertinent to pain management are also worthwhile additions to the text. Each chapter begins with a brief explanation of the mechanism of action followed by a synopsis of specific agents in each class. The inclusion of NNT (the number needed to treat) is an excellent choice; however, there are notable exclusions, including no mention of hydromorphone and the absence of an opioid conversion table, particularly in the context of discussing opioid rotations.

This text can serve as a refresher on various topics for those more knowledgeable about pain management; however, this is where the limitations of a short text on such a broad topic become evident. As one would expect, this is by no means an all-inclusive text on the included topics; this is evident, for example, in the chapter regarding the sacroiliac joint in which only two of the five provocative maneuvers for the joint are outlined. As a result, the information in this text would not suffice for those studying for their Royal College Examination in Anesthesia, and they may be better served by a more complete text on the topic. Additionally, while this text may serve as a quick refresher for practitioners already practicing pain management, it does not serve to provide 
enough detail for adequate clinical management of patient care.

Although this text may not be sufficiently detailed for procedural or clinical use and may lack enough depth for experts in pain management, it does serve as a superb introduction with relevant information for those new to the topic.

Conflicts of interest None declared. 